\title{
Identification and potential mechanisms of a 4-IncRNA signature that predicts prognosis in patients with laryngeal cancer
}

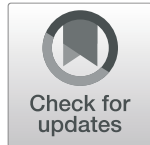

Guihai Zhang $^{1 *+} \mathbb{B}$, Erxi Fan ${ }^{2+}$, Qiuyue Zhong ${ }^{2}$, Guangyong Feng ${ }^{1}$, Yu Shuai ${ }^{2}$, Mingna Wu ${ }^{2}$, Qiying Chen ${ }^{2}$ and Xiaoxia Gou ${ }^{1 *}$

\begin{abstract}
Purpose: This study aimed to describe the use of a novel 4-IncRNA signature to predict prognosis in patients with laryngeal cancer and to explore its possible mechanisms.

Methods: We identified IncRNAs that were differentially expressed between 111 tumor tissue samples and 12 matched normal tissue samples from The Cancer Genome Atlas Database (TCGA). We used Cox regression analysis to identify IncRNAs that were correlated with prognosis. A 4-IncRNA signature was developed to predict the prognosis of patients with laryngeal cancer. The receiver operating characteristic (ROC) curves and area under the curve (AUC) were used to verify the validity of this Cox regression model, and an independent prognosis analysis was used to confirm that the 4-IncRNA signature was an independent prognostic factor. Furthermore, the function of these IncRNAs was inferred using related gene prediction and Gene ontology $(\mathrm{GO})$ enrichment analysis in order to clarify the possible mechanisms underlying their predictive ability.

Results: In total, 214 differentially expressed IncRNAs were identified, and a 4-IncRNA signature was constructed using Cox survival analysis. The risk coefficients in the multivariate Cox analysis revealed that LINC02154 and MNX1-AS1 are risk factors for laryngeal cancer, whereas MYHAS and LINC01281 appear to be protective factors. The results of a functional annotation analysis suggested that the mechanisms by which these IncRNAs influence prognosis in laryngeal cancer may involve the extracellular exosome, the Notch signaling pathway, voltage-gated calcium channels, and the Wht signaling pathway.

Conclusion: We identified a novel 4-IncRNA signature that can predict the prognosis of patients with laryngeal cancer and that may influence the prognosis of laryngeal cancer by regulating immunity, tumor apoptosis, metastasis, invasion, and other characteristics through the Notch signaling pathway, voltage-gated calcium channels, and the Wnt signaling pathway.
\end{abstract}

Keywords: Long noncoding RNA, Laryngeal cancer, TCGA, Prognosis signature, Risk score

\footnotetext{
* Correspondence: zghzhuhai@163.com; angela7695@163.com

${ }^{+}$Guihai Zhang and Erxi Fan contributed equally to this work.

${ }^{1}$ Department of Head and Neck Oncology, Affiliated Hospital of Zunyi

Medical University, Zunyi 563000, Guizhou Province, People's Republic of

China

Full list of author information is available at the end of the article
}

(c) The Author(s). 2019 Open Access This article is distributed under the terms of the Creative Commons Attribution 4.0 International License (http://creativecommons.org/licenses/by/4.0/), which permits unrestricted use, distribution, and reproduction in any medium, provided you give appropriate credit to the original author(s) and the source, provide a link to the Creative Commons license, and indicate if changes were made. The Creative Commons Public Domain Dedication waiver (http://creativecommons.org/publicdomain/zero/1.0/) applies to the data made available in this article, unless otherwise stated. 


\section{Introduction}

Laryngeal cancer is one of the most common malignant head and neck tumors, with an estimated incidence of approximately 11,150 new cases in the United States in 2018, resulting in 3710 deaths. This cancer accounts for approximately $0.8 \%$ of all new cancer cases, and the mortality rate is nearly $0.6 \%$. Accumulating studies suggest that the main risk factors for laryngeal cancer include tobacco, human papillomavirus infection, laryngopharyngeal reflux, environmental and occupational exposures, and alcohol [1]. Over the past few decades, various strategies have been employed to treat laryngeal cancer, but the prognosis of this disease remains unsatisfactory for patients and physicians [2]. According to 2006-2012 data from the SEER database, the 5-year OS of patients with laryngeal cancer remains as low as $60.7 \%$, with no obvious improvement during the past several decades.

Presently, treatment strategies for laryngeal cancer mainly involve surgery and radiation therapy, as well as chemotherapy and gene therapy. Clinical relapse and poor prognosis are most common in cases of advanced laryngeal cancer with local invasion and distant metastasis. When laryngeal cancer is diagnosed at an early stage, the cure rate can reach $80 \% \sim 90 \%$ [3]. However, a majority of patients are still diagnosed with locally advanced disease or regional nodal metastases, and their survival rates are generally less than 50\% [4-6]. Therefore, there is an urgent need to explore novel indicator for tumor detection with prognostic value.

With the development of molecular biology, increasingly more researchers have begun to investigate methods that could be used to predict the prognosis of laryngeal cancer and develop novel therapeutic strategies. lncRNAs are a class of RNAs that are usually more than 200 nucleotides in length and do not code for proteins [7]. Previous reports have shown that lncRNAs can modulate carcinogenesis and influence the rates of metastasis and invasion in various types of cancer [8-11]. Thus, the potential role and prognostic value of many lncRNAs needs to be further explored.

In our study, we used Cox regression survival analysis to construct a novel 4-lncRNA signature that is predictive of prognosis in laryngeal cancer. The final Cox regression model could be applied in clinical settings to predict the prognosis of patients with laryngeal cancer.

\section{Methods \\ Workflow}

We used a combination of methods in several steps to develop the 4-lncRNA signature and explore the possible mechanisms by which these lncRNAs influence the prognosis of laryngeal cancer (Fig. 1).

\section{Dataset processing and screening of differentially expressed IncRNAs}

We collected samples and clinical materials from the TCGA (https://portal.gdc.cancer.gov/) and selected the HTseq-count. Differentially expressed lncRNAs were selected using edgeR software ( $P$-value $\leq 0.001$ and fold change $\geq 2$ ) [12].

\section{ceRNA network construction and KEGG enrichment}

We used the miRcode database (Jeggari, Marks \& Larsson 2012) to predict lncRNA-miRNA interactions. The corresponding coding genes were then identified using miRDB, miRTarBase, and TargetScan [13-15]. We only chose miRNA-targeted mRNAs present in all three databases and differentially expressed mRNAs to enhance

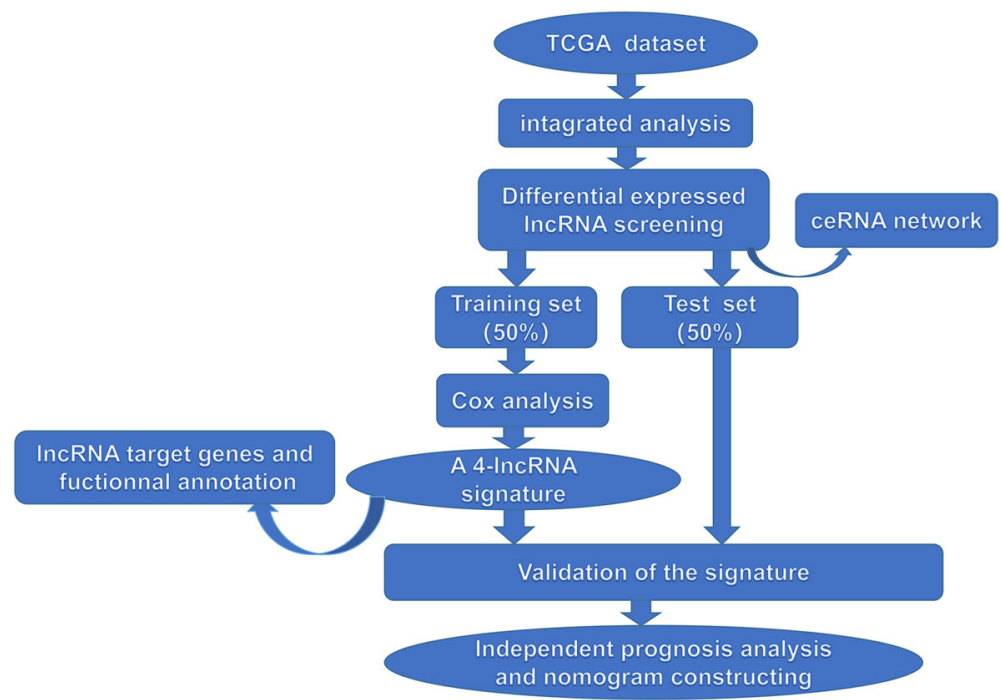

Fig. 1 Main workflow for the study 
the validity of this ceRNA network. Furthermore, the ceRNA network was visualized using Cytoscape v3.6.1 [16].

IncRNA signature identification and risk score calculation To identify a multi-lncRNA signature with good predictive performance for prognosis, we randomly divided the samples into a training set and a test set. Differentially expressed lncRNAs that were correlated with patients' OS in the training set were screened using univariate Cox regression analysis. lncRNAs with a $P$ value $<0.05$ were considered as candidate variables, and the lncRNAs with the lowest Akaike information criterion (AIC) values were retained in the final signature. The risk coefficients for these lncRNAs were calculated using a multivariable Cox proportional hazards model implemented with the "survival" and "survminer" $\mathrm{R}$ packages. Detailed R code is presented in the Additional file 2. We used the following risk formula: riskscore $=\sum \beta \ln \mathrm{c} R N A \mathrm{i} \times$ Exp ln $\mathrm{CRNA \textrm {i }} \quad(\mathrm{i}=1-\mathrm{n})$, where $\beta_{\text {IncRNAi }}$ indicates the coef and ExplncRNAi indicates the expression level in patients with laryngeal cancer. All patients were divided into high-risk and low-risk groups according to their median risk score. We used the Kaplan-Meier method to analyze the OS of the two groups and the prediction value of the model was verified by plotting receiver operating characteristic curves (ROCs) for the training set, the test set, and the entire set. All data were processed and analyzed using $\mathrm{R}$ (version 3.5.1) and Perl 5 version 24 [17].

\section{Independent prognosis analysis and nomogram construction}

The prognostic value of clinical variables and the risk score calculated using the lncRNA signature for the OS of laryngeal patients was initially assessed in univariate Cox proportional hazards regression analyses. Subsequently, each variable was evaluated in a multivariate Cox proportional hazards regression analysis. Furthermore, a nomogram to predict patients' prognosis was constructed using R packages "Hmisc", "lattice", "Formula", "ggplot2", "foreign" and "rms".

\section{Prediction analysis of IncRNA-related genes and gene ontology (GO) functional annotation}

We identified genes related to the lncRNAs in the predictive signature using the Multi Experiment Matrix (MEM) database (https://biit.cs.ut.ee/mem/). We then uploaded these genes to the David website (https://david.ncifcrf.gov) to perform GO functional enrichment analysis, with $P<0.05$ as the cutoff value. Finally, the results were visualized using Cytoscape v3.6.1 [16].
Table 1 Characteristics of the datasets

\begin{tabular}{|c|c|}
\hline Variable & Case, $n$ \\
\hline \multicolumn{2}{|l|}{ Age } \\
\hline$\leq 49$ & 11 \\
\hline$>49$ & 101 \\
\hline \multicolumn{2}{|l|}{ Gender } \\
\hline male & 92 \\
\hline female & 20 \\
\hline \multicolumn{2}{|l|}{ T stage } \\
\hline $\mathrm{T} 1+\mathrm{T} 2$ & 20 \\
\hline $\mathrm{T} 3+\mathrm{T} 4$ & 79 \\
\hline TX & 11 \\
\hline NA & 2 \\
\hline \multicolumn{2}{|c|}{ Lymph node status } \\
\hline NO & 40 \\
\hline N1-3 & 53 \\
\hline NX & 17 \\
\hline NA & 2 \\
\hline \multicolumn{2}{|l|}{ Metastasis } \\
\hline MO & 41 \\
\hline $\mathrm{M} 1$ & 1 \\
\hline$M X$ & 8 \\
\hline NA & 62 \\
\hline
\end{tabular}

\section{Results}

Characteristics of the datasets

We collected 123 samples from 112 patients with laryngeal carcinoma from the TCGA, consisting of 111 laryngeal tumor tissue samples and 12 matched normal tissue samples. The clinical data and materials were collected from 92 men and 20 women, of whom 43 had died. The age of patients with laryngeal cancer ranged from 38 to 83 years, and their survival post-diagnosis ranged from 2 to 6417 days. More specific characteristics are shown in Table 1.

\section{Differential expression of IncRNAs}

A total of 214 differentially expressed lncRNAs were screened from the TCGA datasets (fold-change $\geq 2, P<$ 0.001), including 54 downregulated lncRNAs and 160 upregulated lncRNAs. Figure 2 illustrates the upregulated and downregulated genes using a volcano map and a heatmap.

\section{ceRNA network construction and KEGG enrichment analysis}

We used 214 differentially expressed lncRNAs retrieved from the mircode database and identified 1597 pairs of interacting lncRNAs and miRNAs using the 

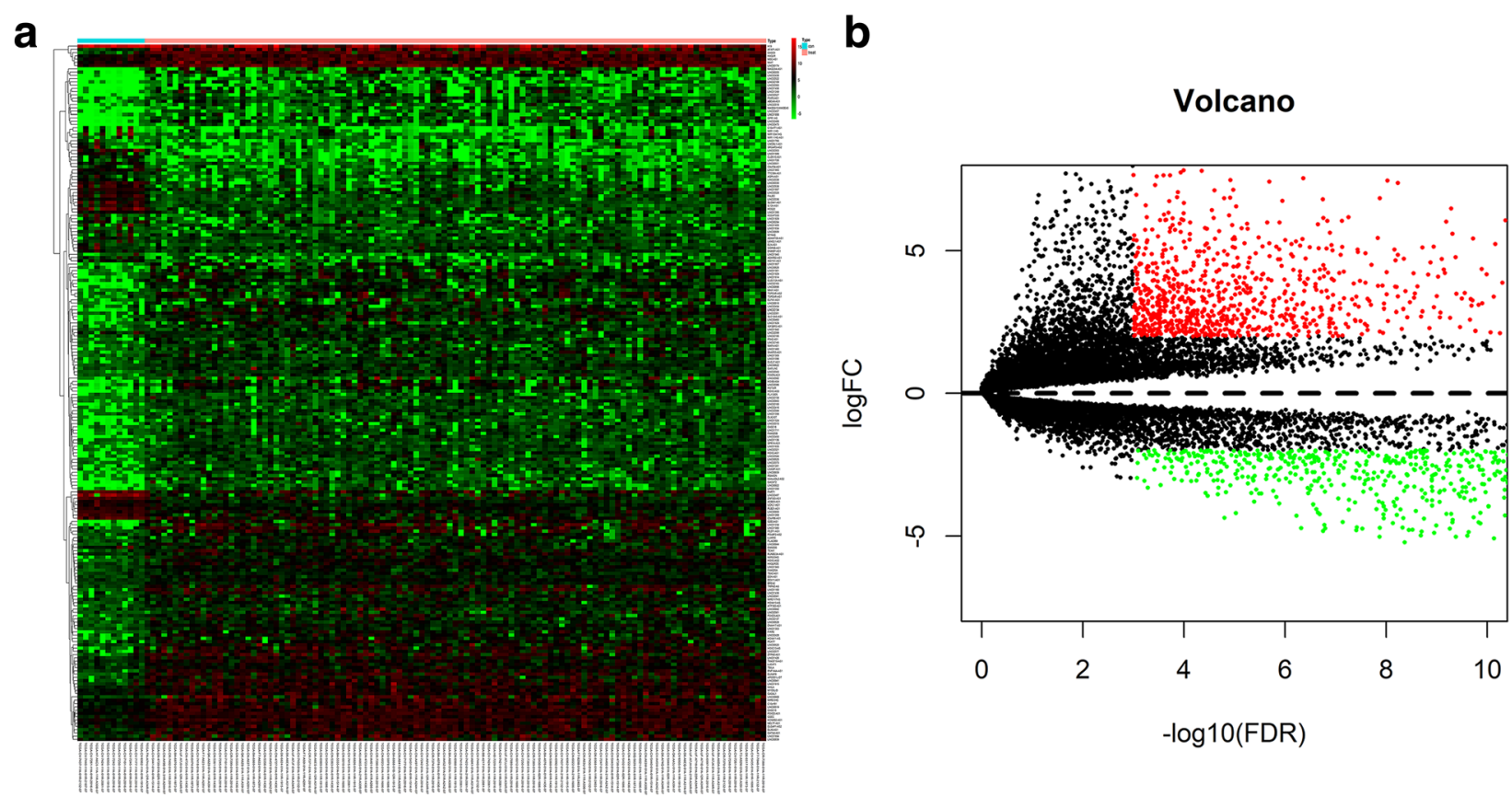

Fig. 2 a Heatmap of differentially expressed IncRNAs. We conducted gene expression profiling to identify IncRNAs that were differentially expressed between 111 tumor tissue samples and 12 normal tissue samples. b Volcano plot of differentially expressed IncRNAs. Each red dot represents an upregulated gene, and each green dot represents a downregulated gene

Perl program. In all, we identified a total of 28 lncRNA nodes, 18 miRNA nodes and 40 mRNA nodes as differentially expressed profiles in the ceRNA network (Additional file 1), but none of the plausible ceRNAs were associated with laryngeal cancer survival.

\section{Identification of a 4-IncRNA signature}

We randomly divided 110 samples and the corresponding clinical data into a training set $(n=55)$ and a test set $(\mathrm{n}=55)$. In the training set, 214 differentially expressed lncRNAs were screened using univariate survival analysis to identify those that best correlated with prognosis. Multivariate Cox regression analysis was then used to develop a 4-lncRNA signature that is an independent predictor of 5 -year OS $(P<0.05)$. The 4 lncRNAs included in this signature were LINC02154, LINC01281, MYHAS, and MNX1-AS1. The risk coefficients suggested that LINC02154 and MNX1-AS1 are risk factors for laryngeal cancer (coef $>0)$, whereas MYHAS and
LINC01281 appeared to be protective factors $($ coef $<0)$ (Table 2). This 4-lncRNA signature is of great importance for prognostic evaluation in laryngeal cancer. The risk score of each patient was calculated based on the following formula: riskscore $=\sum \beta \ln \mathrm{c} R N A \mathrm{i} \times \operatorname{Exp} \ln$ cRNAi $(\mathrm{i}=1-4)=0.204491007100773 \times \operatorname{Exp}_{\text {LINC02154 }}+$ $-0.32288924590121 \times$ Exp MYHAS $+0.167587835674659 \times$ Exp $_{M N X 1-A S 1}+-0.381674952789156 \times \quad E_{\text {Xxp }}$ LINC2181. Higher risk scores represent worse clinical prognosis. Accordingly, we divided patients into high- and low-risk groups depending on their median risk score to assess the score's ability to accurately predict survival in a Cox regression model. The survival curve revealed a significant difference in OS between the high- and low-risk groups, confirming that the risk score could be an independent predictor of OS (Fig. 3).

\section{Validation of cox regression model}

The AUC of the ROC curve was 0.822 in the training set, 0.741 in the test set, and 0.773 in the entire set.

Table 2 A 4-IncRNA signature identified by multivariate Cox regression analysis

\begin{tabular}{llllll}
\hline id & coef & exp (coef) & se (coef) & $z$ & Pr $(>|z|)$ \\
\hline LINC02154 & 0.204491007 & 1.226900422 & 0.098957801 & 2.066446566 & 0.038786333 \\
MYHAS & -0.322889246 & 0.724054042 & 0.172769207 & -1.868905064 & 0.061636024 \\
MNX1-AS1 & 0.167587836 & 1.182449147 & 0.109102613 & 1.536057025 & 0.124524395 \\
LINC01281 & -0.381674953 & 0.682716932 & 0.163786433 & -2.330320927 & 0.019789195 \\
\hline
\end{tabular}


a

Hazard ratio

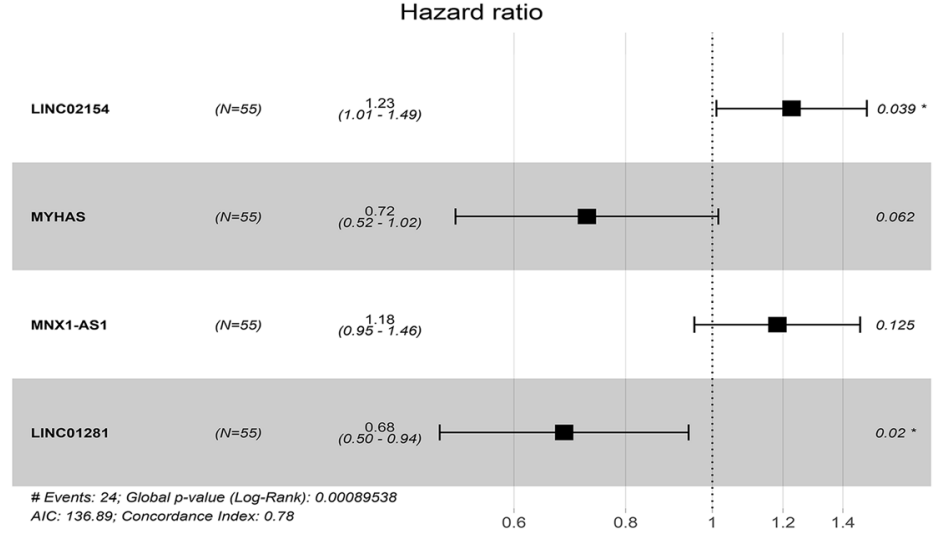

b

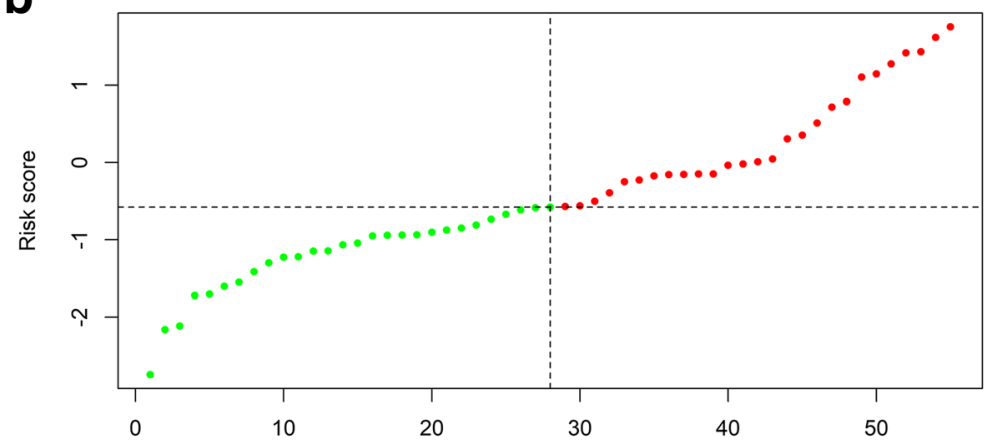

C

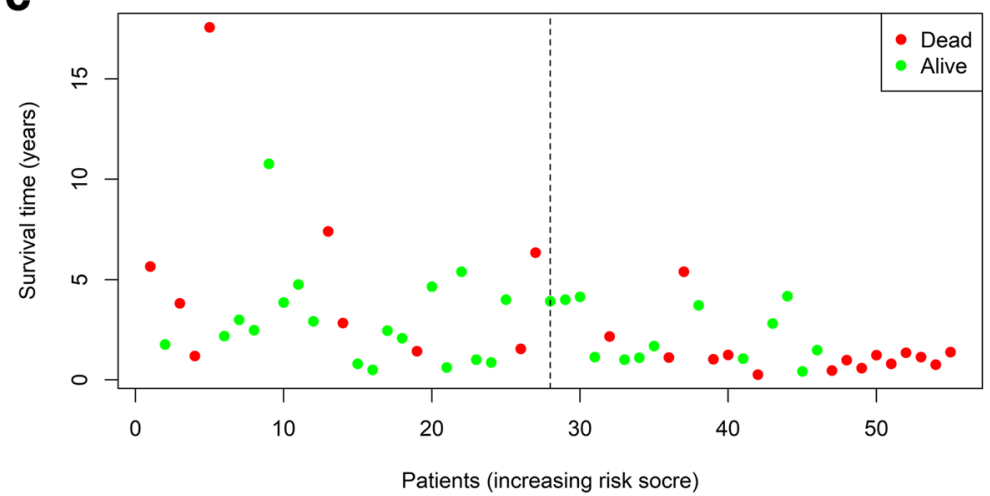

Fig. 3 a Forest map of the 4-IncRNA signature. $\mathbf{b}$ IncRNA risk score. c Distribution of patient survival status and survival time

Moreover, there were significant differences in OS between the high- and low-risk groups in all three sets, demonstrating that this signature performs well as a predictor of prognosis in laryngeal cancer patients (Fig. 4). Figure 5 shows a heatmap illustrating the distribution of risk scores across all patients for each gene.

\section{The 4-IncRNA signature is an independent predictor of laryngeal cancer prognosis}

Univariate and multivariate Cox regression analyses were used to investigate whether the 4-IncRNA signature was an independent predictor of prognosis among laryngeal cancer patients, using the risk score and other clinicopathological data, including age, sex, and stage. In the univariate Cox regression, the risk score and sex were significantly correlated with patients' overall survival. In the multivariate Cox regression analysis, after adjusting for age and other covariates, the risk score based on the 4-lncRNA signature remained an independent predictor (Table 3). Moreover, a nomogram was constructed that integrated the 4-IncRNA signature risk score and other clinicopathological characteristics such as age, sex, and stage. A higher total number of points on the nomogram indicates a worse prognosis (Fig. 6). 


\section{a}

Training set

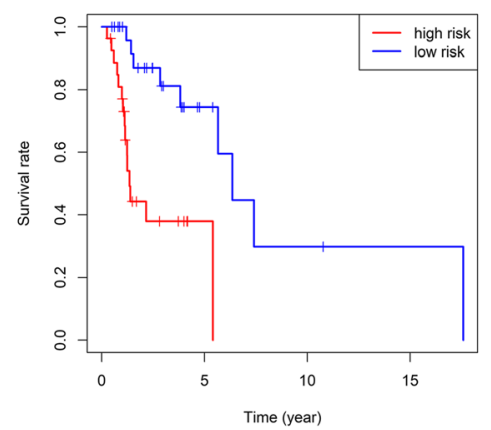

b

Test set

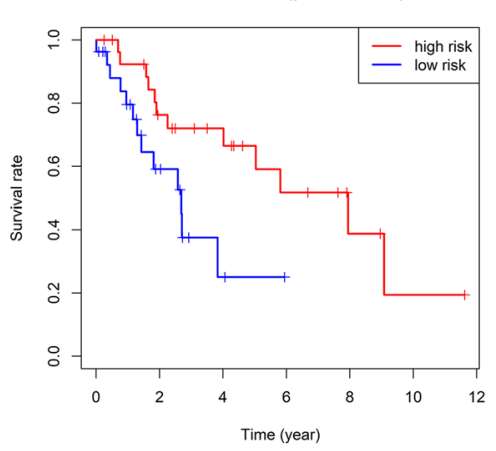

C

Entire set

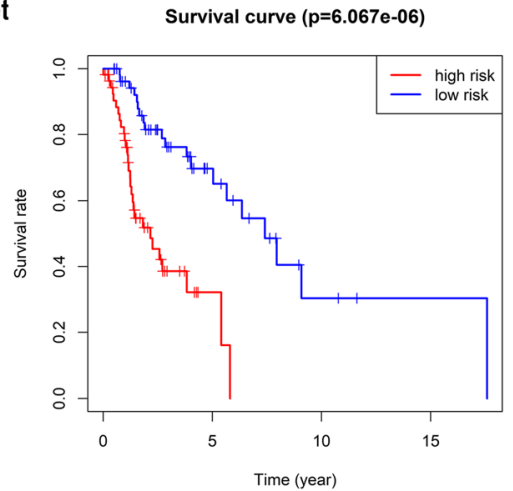

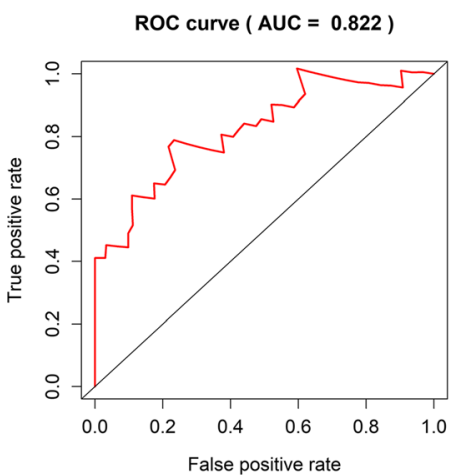

ROC curve $(A U C=0.741)$

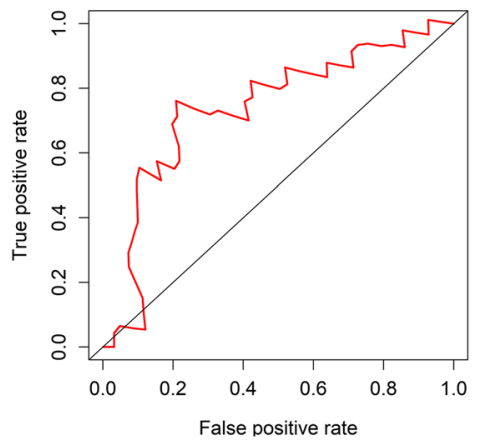

ROC curve $(A U C=0.773)$

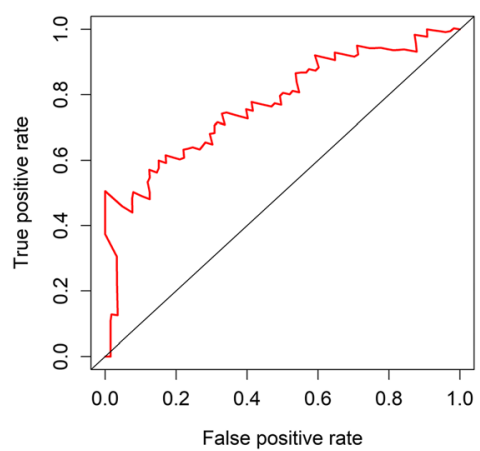

Fig. 4 Validation of the 4-IncRNA signature. The area under the ROC was 0.822 in the training set, 0.741 in the test set, and 0.773 in the entire set. There were significant differences in OS between the high- and low-risk groups in all three sets. The results show that the 4-IncRNA signature performed well in predicting the prognosis of laryngeal cancer. a Training set, $\mathbf{b}$ Test set, $\mathbf{c}$ Entire set

\section{Prediction of IncRNA-related genes and functional annotation}

We identified genes related to the signature lncRNAs using the MEM database. The top 200 genes that most closely related to LINC01281 and MNX1-AS1 are listed in Additional file 3. We were unable to search for genes related to LINC02154 and MYHAS in MEM. The relationship between the lncRNAs and the related genes and the GO functional annotation analysis results are shown in Fig. 7.

\section{Discussion}

Laryngeal cancer is a malignant head and neck squamous cell carcinoma [18]. Currently, several therapeutic strategies such as surgery, radiation therapy and chemotherapy are employed in clinical treatment. Owing to the development of treatment strategies for this malignancy, the survival rates of laryngeal cancer patients have improved to some extent [19]. However, the prognosis of patients with advanced laryngeal cancer has not improved over the past several decades. In fact, metastasis 


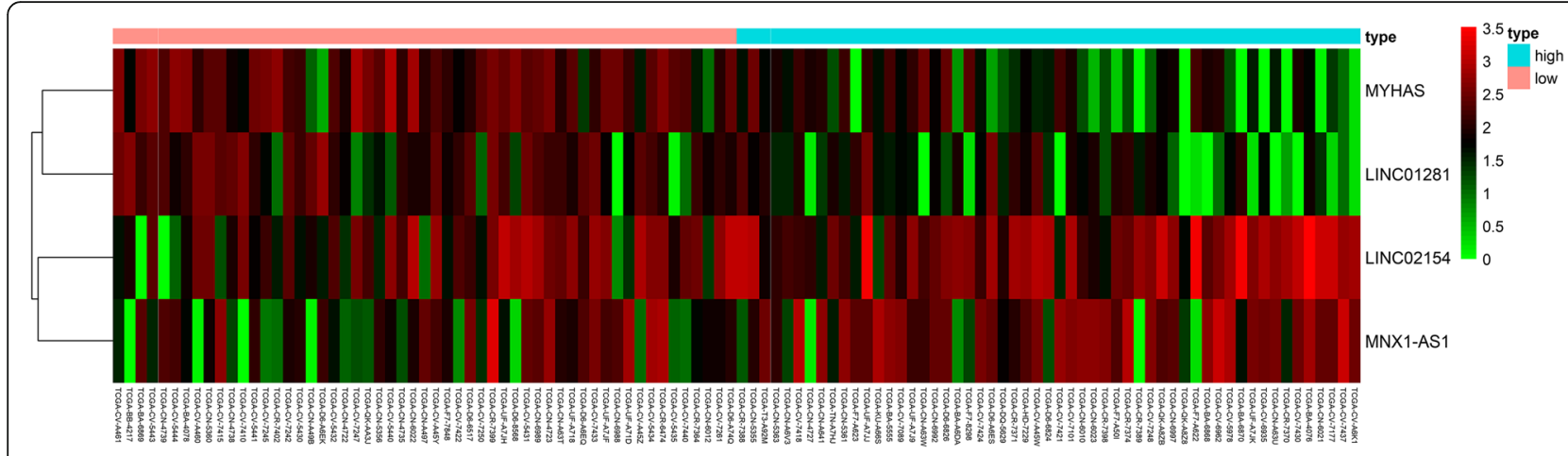

Fig. 5 Heatmap of the 4-IncRNA signature in the TCGA datasets. Each column represents a sample, and each row represents one of the 4 IncRNAs. The expression levels of the 4 IncRNAs are shown in different colors, varying from orange to blue with increasing risk

and recurrence are the main causes of poor prognosis and decrease the overall survival of laryngeal cancer patients by more than $50 \%$ [20]. Because of the non-specific symptoms of laryngeal cancer, the disease is often diagnosed late, when it is already in advanced stages, resulting in delayed treatment [21]. Therefore, there is an urgent need to explore novel and effective prognostic indicators in laryngeal cancer. The current development of bioinformatics technologies provides powerful, high-throughput tools to screen molecular biomarkers and indicators of prognosis. Recently, increasingly more researchers have focused on the clinical significance of lncRNAs on the prognosis of multiple types of tumors; these lncRNAs have tissue-specific expression patterns and play crucial roles in the progression of diseases. For instance, Zhang $\mathrm{K}$ et al. [22] observed that the lncRNA AOC4P promotes tumourigenesis and progression partly through epithelial-mesenchymal transition, thereby promoting a poor prognosis in gastric cancer. The study by Bo $\mathrm{H}$ et al. [23] showed that the expression level of the lncRNA AFAP1-AS1 may be involved in the development of cervical cancer, and Beltrán-Anaya FO et al. [24] confirmed that the low expression of LncKLHDC7B was associated with poor prognosis in patients with breast cancer.

In our study, we constructed a 4-lncRNA risk model that predicts prognosis in laryngeal cancer. MYHAS and LINC01281 were associated with a better prognosis among laryngeal cancer patients. Therefore, this novel 4-lncRNA signature could be used to predict OS for laryngeal cancer patients. Furthermore, using the MEM database and

Table 3 Univariate and multivariable Cox proportional-hazards regression analysis on OS

\begin{tabular}{|c|c|c|c|c|c|c|}
\hline \multicolumn{4}{|c|}{ Univariate Cox } & \multicolumn{3}{|c|}{ multivariate Cox } \\
\hline Variables & $\mathrm{HR}$ & $95 \%$ IC of HR & $P$-value & $\mathrm{HR}$ & $95 \%$ IC of HR & $P$-value \\
\hline Sex & 0.30 & $0.132-0.701$ & 0.005 & 0.376 & $0.154-0.917$ & 0.032 \\
\hline Age & 1.00 & $0.959-1.046$ & 0.935 & 0.972 & $0.931-1.014$ & 0.190 \\
\hline Stage & 0.91 & $0.560-1.466$ & 0.687 & 1.024 & $0.606-1.731$ & 0.929 \\
\hline riskScore & 2.39 & $1.698-3.370$ & 0.000 & 2.522 & $1.727-3.685$ & 0.000 \\
\hline
\end{tabular}

functional enrichment analysis, we hypothesized possible mechanisms by which lncRNAs influence the prognosis of patients with laryngeal cancer. According to the functional annotation results, genes related to MNX1-AS1 significantly enrich on GO terms 0007219 (Notch signaling pathway) and 0070062 (extracellular exosome). The Notch signaling pathway is evolutionarily conserved in mammals and plays an important role in cell development and differentiation. In recent years, increasing evidence has shown that Notch is associated with tumor development. Aberrant activation of the Notch signaling pathway has been found in many different solid tumors, and it has been found to induce cell proliferation, metastasis and epithelial-mesenchymal transition [25]. The exosome has been found to play important roles in many physiological and pathological processes such as antigen presentation in immunization, tumor growth and migration, and repair of tissue damage. Exosomes secreted by various cells have different components and functions and they are used as biomarkers for disease diagnosis. Exosomes possess a lipid bilayer membrane structure that can protect the coated material well and can target specific cells or tissues, so they constitute a well-targeted delivery system [26]. Generally, the possible mechanisms by which MNX1-AS1 may lead to poor prognosis are mainly related to immune response and the Notch signaling pathway. LINC01281 enriched on GO terms 0005891 (voltage-gated calcium channel complex) and 0030178 (negative regulation of Wnt signaling pathway). Voltage-gated calcium channels (VGCCs) are well documented to play roles in cell proliferation, migration, and apoptosis. Chih-Yang Wang et al. showed that many calcium channel subunits are overexpressed and likely involved in the development of various types of cancers. The observed overexpression of CACNA1A, CACNA1C, and CACNA1D suggests that they are likely targets for cancer treatment, as blockage or partial inhibition of their expression could help to modulate the progression of metastatic disease [27]. Many previous reports revealed that the Wnt signaling pathway is a highly conserved pathway controlling 


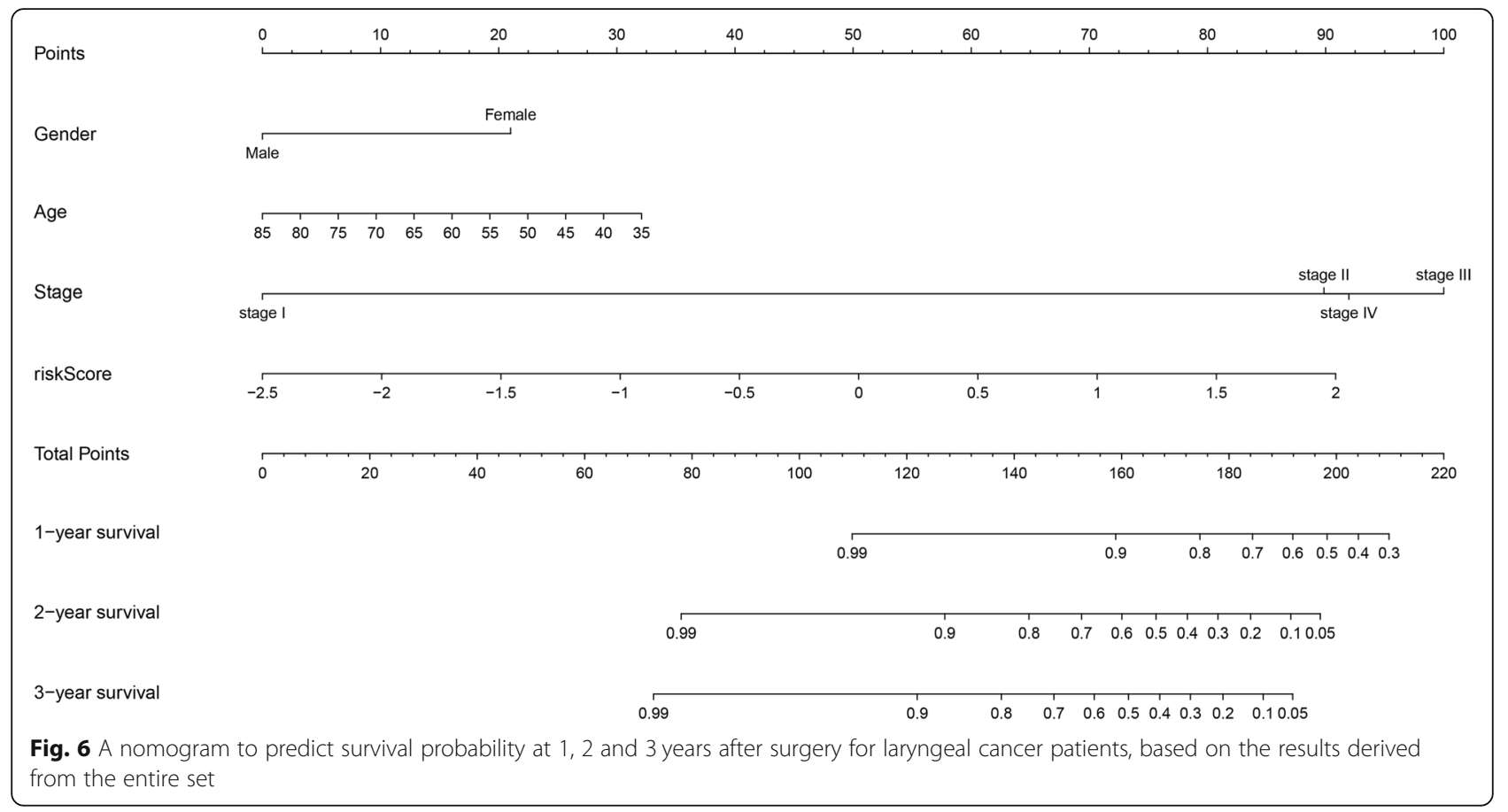

cell growth, differentiation, apoptosis and self-renewal. This signaling pathway is often abnormally activated in tumor development and progression, and it can enhance or antagonize other signaling pathways to regulate tumor proliferation, migration, and invasion [28-30]. Based on our prediction and functional annotation analysis of genes related to our selected IncRNAs, we speculate that these lncRNAs may influence the prognosis of laryngeal cancer through mechanisms involving immune response, the Notch signaling pathway, voltage-gated calcium channels, and negative regulation of the Wnt signaling pathway.

In summary, we constructed a novel 4-lncRNA signature that can be used to predict the prognosis of patients with laryngeal cancer and hypothesized possible underlying mechanisms. However, there are some limitations in our study. First, we obtained data only from the
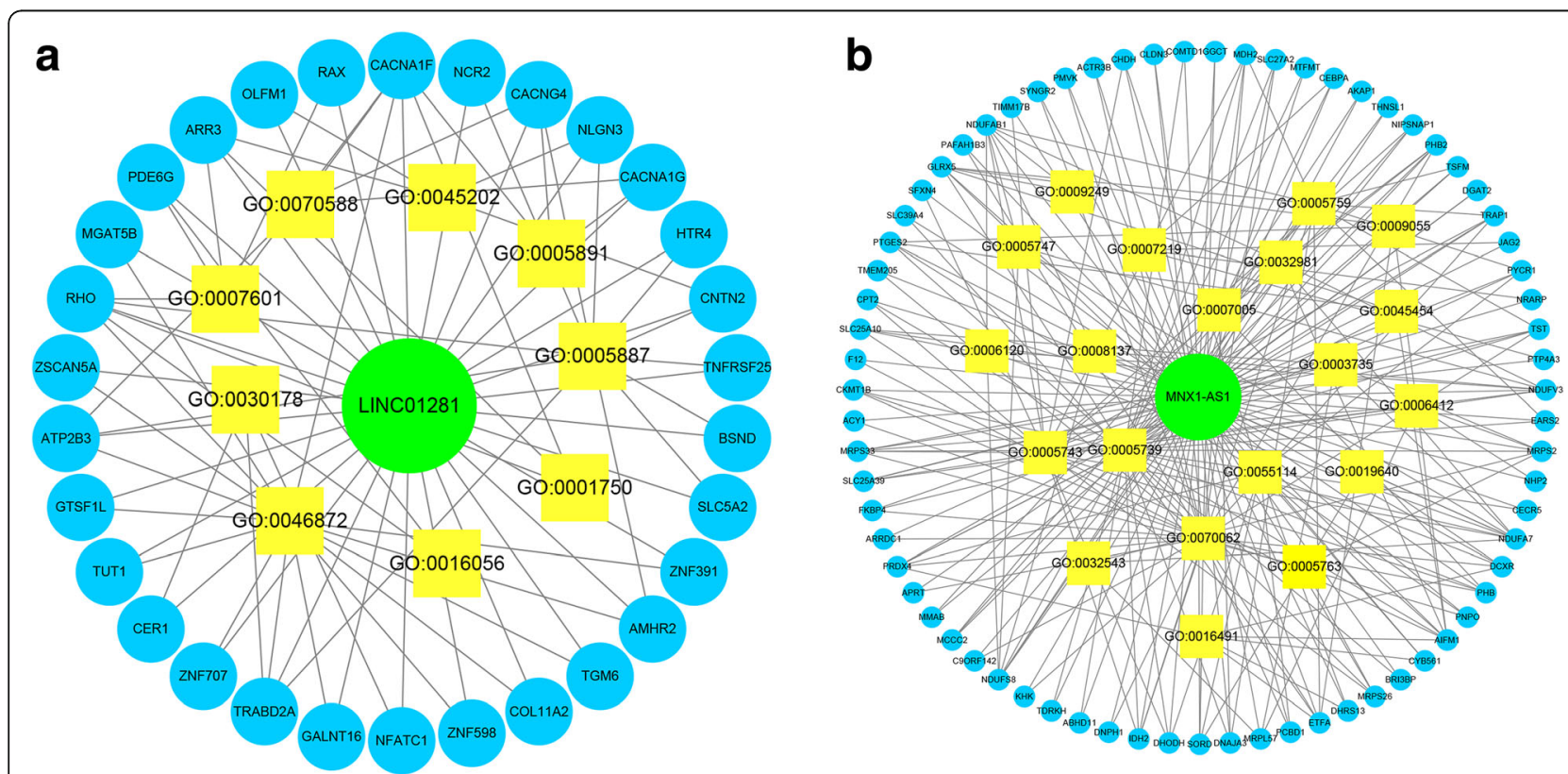

Fig. 7 Relationship between the IncRNAs and related genes, and GO functional annotation analysis results. a The related genes of LINC01281 and the GO functions that they enriched on. $\mathbf{b}$ The related genes of MNX1-AS1 and the GO functions that they enriched on 
TCGA datasets, and the sample size is not very large. Second, we provide only preliminary hypotheses on the possible mechanisms by which the selected IncRNAs impact prognosis. Further studies should be conducted to clarify the overall mechanism by which these lncRNAs influence the prognosis of laryngeal cancer. Finally, all of the above results need to be verified in basic experiments and clinical trials.

\section{Conclusion}

We constructed a novel 4-lncRNA signature that can predict the prognosis of patients with laryngeal cancer. The mechanisms underlying this predictive relationship may involve the extracellular exosome, the Notch signaling pathway, voltage-gated calcium channels, and the Wnt signaling pathway. These findings may provide a better understanding of the prognostic value of lncRNAs and related mechanisms.

\section{Additional files}

Additional file 1: ceRNA network. Competitive endogenous RNA (ceRNA) regulation network of differentially expressed IncRNAs in laryngeal cancer. (PDF $7834 \mathrm{~kb})$

Additional file 2: $\mathrm{R}$ language code. $\mathrm{R}$ language code of Multivariate Cox regression analysis to develop a 4-IncRNA signature. (TXT $1 \mathrm{~kb}$ )

Additional file 3: IncRNA-related genes. Top 200 IncRNA-related genes predicted by MEM. (PDF $7558 \mathrm{~kb}$ )

\section{Abbreviations}

AUC: area under the curve; GO: Gene ontology; KEGG: Kyoto Encyclopedia of Genes and Genomes; MEM: Multi Experiment Matrix; OS: Overall survival; ROC: receiver operating characteristic; TCGA: The Cancer Genome Atlas Database

\section{Acknowledgements}

We appreciate the generosity of TCGA network for sharing the huge amount of data.

\section{Authors' contributions}

GZ conceptualized the main idea of the project. EF and QZ carried out the data processing, coding, and computational. GZ, XG, GF, YS, MW, QC designed the study and supervised the project. GZ, EF, QZ, GF, YS, MW, QC and $X G$ wrote the paper. All authors read and approved the final manuscript.

\section{Funding}

This study was financially supported by National Natural Science Foundation of China (No. 81260370)

\section{Availability of data and materials}

The datasets supporting the results of this article are publicly available at the TCGA (https://portal.gdc.cancer.gov/).

\section{Ethics approval and consent to participate}

This study was approved by the Affiliated Hospital of Zunyi Medical University institutional review board (IRB). The data were obtained from TCGA (https://portal.gdc.cancer.gov/). Informed consent has been obtained from all individual participants included in the study was approved by the Institutional review Board.

\section{Consent for publication}

Not applicable.

\section{Competing interests}

The authors declare that they have no competing interests.

\section{Author details}

${ }^{1}$ Department of Head and Neck Oncology, Affiliated Hospital of Zunyi Medical University, Zunyi 563000, Guizhou Province, People's Republic of China. 'Zunyi Medical University, Zunyi 563000, Guizhou Province, People's Republic of China.

Received: 26 March 2019 Accepted: 31 July 2019

Published online: 15 August 2019

\section{References}

1. Obid R, Redlich M, Tomeh C. The treatment of laryngeal Cancer. Oral Maxillofac Surg Clin North Am. 2019;31:1-11.

2. García Lorenzo J, Montoro Martínez V, Rigo Quera A, et al. Modifications in the treatment of advanced laryngeal cancer throughout the last 30 years. Eur Arch Otorhinolaryngol. 2017;274:3449-55.

3. Forastiere AA, Ismaila N, Wolf GT, et al. Use of larynx-preservation strategies in the treatment of laryngeal Cancer: American Society of Clinical Oncology clinical practice guideline update summary. J Oncol Pract. 2018;14:123-8.

4. Ben-Dayan MM, OW TJ, Belbin TJ. Nonpromoter methylation of the CDKN2A gene with active transcription is associated with improved locoregional control in laryngeal squamous cell carcinoma. Cancer Med. 2017;6:397-407.

5. Wong N, Khwaja SS, Baker CM, et al. Prognostic microRNA signatures derived from the Cancer genome atlas for head and neck squamous cell carcinomas. Cancer Med. 2016;5:1619-28.

6. Huang GJ, Luo MS, Chen GP, et al. miRNA-mRNA crosstalk in laryngeal squamous cell carcinoma based on the TCGA database. Eur Arch Otorhinolaryngol. 2018;275:751-9.

7. Wang X, Zhou J, Xu M, et al. A 15-IncRNA signature predicts survival and functions as a ceRNA in patients with colorectal cancer. Cancer Manag Res. 2018;10:5799-806

8. Wang, et al. LncRNA MIR31HG targets HIF1A and P21 to facilitate head and neck cancer cell proliferation and tumorigenesis by promoting cell-cycle progression. Mol Cancer. 2018;17:162.

9. Ma Y, Luo T, Dong D, et al. Characterization of long non-coding RNAs to reveal potential prognostic biomarkers in hepatocellular carcinoma. Gene. 2018;663:141-56.

10. $\quad$ Yang H, Liu P, Zhang J, Peng $X$, et al. Long noncoding RNA MIR31HG exhibits oncogenic property in pancreatic ductal adenocarcinoma and is negatively regulated by miR-193b. Oncogene. 2016;35:3647-57.

11. Montes M, Nielsen MM, Maglieri G, et al. The IncRNA MIR31HG regulates p16(INK4A) expression to modulate senescence. Nat Commun. 2015;6:6967.

12. Robinson MD, Mccarthy DJ, Smyth GK. edgeR: a Bioconductor package for differential expression analysis of digital gene expression data. Bioinformatics. 2010;26(1):139-40.

13. Wang $X$. Improving microRNA target prediction by modeling with unambiguously identified microRNA-target pairs from CLIP-ligation studies. Bioinformatics. 2016:32(9):1316-22.

14. Wong $N$, Wang $X$. miRDB: an online resource for microRNA target prediction and functional annotations. Nucleic Acids Res. 2015;43(D1):D146-52

15. Chou CH, Chang NW, Shrestha S, et al. miRTarBase 2016: updates to the experimentally validated miRNA-target interactions database. Nucleic Acids Res. 2016:44(D1):D239-47.

16. Shannon P, Markiel A, Ozier O, et al. Cytoscape: a software environment for integrated models of biomolecular interaction networks. Genome Res. 2003; 13(11):2498-504.

17. Yu G, Wang LG, Han Y, He QY, Qy H. clusterProfiler: an R package for comparing biological themes among gene clusters. OMICS. 2012;16(5):284-7.

18. Bolzoni Villaret A, Barbieri D, Peretti G, et al. Angiogenesis and lymphangiogenesis in early-stage laryngeal carcinoma: prognostic implications. Head Neck. 2013;35:1132-7.

19. Pfister DG, Laurie SA, Weinstein GS. American Society of Clinical Oncology clinical practice guideline for the use of larynx-preservation strategies in the treatment of laryngeal cancer. J Clin Oncol. 2006;24:3693-704.

20. Li C, Chen L, Wang J, et al. Expression and clinical significance of cathepsin $B$ and stefin a in laryngeal cancer. Oncol Rep. 2001;26:869-75.

21. Zeng W, Li Y, Lu E, Ma M, et al. CYP1A1 rs1048943 and rs4646903 polymorphisms associated with laryngeal cancer susceptibility among Asian populations: a meta-analysis. J Cell Mol Med. 2016;20(2):287-93. 
22. Zhang K, Lu C, Huang X, Cui J, et al. Long noncoding RNA AOC4P regulates tumor cell proliferation and invasion by epithelial-mesenchymal transition in gastric cancer. Ther Adv Gastroenterol. 2019;12:1-14.

23. Bo H, Fan L, Gong Z, et al. Upregulation and hypomethylation of IncRNA AFAP1-AS1 predicts a poor prognosis and promotes the migration and invasion of cervical cancer. Oncol Rep. 2019:41:2431.

24. Beltrán-Anaya FO, Romero-Córdoba S, Rebollar-Vega R, Arrieta O, et al. Expression of long non-coding RNA ENSG00000226738 (LncKLHDC7B) is enriched in the immunomodulatory triple-negative breast cancer subtype and its alteration promotes cell migration, invasion, and resistance to cell death. Mol Oncol. 2019;13:909.

25. Li L, Tang P, Li S, et al. Notch signaling pathway networks in cancer metastasis: a new target for cancer therapy. Med Oncol (Northwood, London, England). 2017;34(10):180.

26. Hoof, V.\& A. Exosome-mediated recognition and degradation of mrnas lacking a termination codon. Science. 2002;295(5563):2262-4.

27. Wang CY, Lai MD, Phan NN, et al. Meta-analysis of public microarray datasets reveals voltage-gated calcium gene signatures in clinical Cancer patients. PLoS One. 2015;10(7):e0125766.

28. Abbaszadegan MR, Riahi A. Forghanifard MM.WNT and NOTCH signaling pathways as activators for epidermal growth factor receptor in esophageal squamous cell carcinoma. Cell Mol Biol Lett. 2018;23:42.

29. Kleszcz R, Szymańska A, Krajka-Kuźniak V, et al. Inhibition of CBP/B-catenin and porcupine attenuates Wnt signaling and induces apoptosis in head and neck carcinoma cells. Cel Oncol (Dordrecht). 2019;42(4):505.

30. Zhan T, Ambrosi G, Wandmacher AM, et al. MEK inhibitors activate Wnt signalling and induce stem cell plasticity in colorectal cancer. Nat Commun. 2019;10(1):2197.

\section{Publisher's Note}

Springer Nature remains neutral with regard to jurisdictional claims in published maps and institutional affiliations.

Ready to submit your research? Choose BMC and benefit from:

- fast, convenient online submission

- thorough peer review by experienced researchers in your field

- rapid publication on acceptance

- support for research data, including large and complex data types

- gold Open Access which fosters wider collaboration and increased citations

- maximum visibility for your research: over $100 \mathrm{M}$ website views per year

At $\mathrm{BMC}$, research is always in progress.

Learn more biomedcentral.com/submissions 